\title{
Assessment and Comparison between Cervical Retraction Exercise and Muscle Energy Technique on Cervical Joint Position Sense in Individuals with Forward Head Posture
}

\author{
Isha Shah ${ }^{1}$, Parag Kulkarni² ${ }^{2}$ Ajay Kumar ${ }^{3}$ \\ ${ }^{1}$ M.P.T, DPO's NETT College of Physiotherapy, Thane, Maharashtra, India \\ ${ }^{2}$ Assistant Professor, DPO's NETT College of Physiotherapy, Thane. \\ ${ }^{3}$ Principal, DPO's NETT College of Physiotherapy, Thane. \\ Corresponding Author: Isha Shah
}

DOI: https://doi.org/10.52403/ijshr.20220132

\begin{abstract}
Background: Forward Head posture (FHP) one of the most common postural disorders causes a change in the biomechanical movement, affect the length---tension relationship in the cervical muscles, increase muscular activity level and impair cervical proprioception. Cervical Retraction and Muscle Energy Technique (MET) have been proposed to improve joint proprioception.

Objective: To assess and compare the effect between Cervical Retraction and Muscle Energy Technique on cervical joint position sense in individuals with forward head posture using head repositioning test.

Method: This is a comparative study where 60 subjects were selected as per inclusion and exclusion criteria and assigned to Group A $(n=30$; Cervical Retraction) and Group B ( $n=30$; Muscle Energy Technique) respectively. Proprioceptive exercises were given to both the groups. The intervention was given for 2 weeks3 sessions per week. Error in Cervical Joint Position sense was assessed pre and post intervention using Head Repositioning Accuracy test. The data was collected and statistically analysed.

Results: The study showed statistical significant reduction $(\mathrm{p}<0.05)$ in error in cervical joint position sense within the groups as well as between the groups for all the physiological movement (Flexion, Extension, Left Rotation and Right Rotation)
\end{abstract}

Conclusion: Cervical Retraction as well as Muscle Energy Technique can be used to improve Cervical Joint position sense. However, Cervical Retraction is better as compared to Muscle Energy Technique.

Key Words: Forward head posture, Cervical Joint Position sense, Cervical Retraction, Muscle Energy Technique, Head Repositioning Accuracy test.

\section{INTRODUCTION}

Body posture is defined as a state of alignment of the body for a specific amount of time, while an ideal posture can be described as state of maintaining balance in the body using minimal musculoskeletal activity without causing pain or discomfort. ${ }^{1}$ The anterior and posterior cervical musculature forms a sleeve which encompasses and stabilizes the cervical spine in all positions of the head. ${ }^{2}$ Any change in one of these structures can lead to postural imbalance not only locally but also in other muscular chains because of a close relationship between them. ${ }^{2}$ Poor cervical posture is examined to be inefficient, increasing anti-gravity load on cervical structures leading to an abnormal and compensatory activity by them and resulting in pain. ${ }^{2}$ This can instigate changes in the alignment of the spine, leading to improper 
posture, such as a rounded shoulder or forward head posture (FHP). ${ }^{1}$ Forward Head posture (FHP) has been defined as 'any alignment in which the external auditory meatus is positioned anterior to the plumb line through the shoulder joint' ${ }^{3}$ Forward head results in a posture which extends head and upper cervical vertebrae, and the lower cervical vertebrae flex. ${ }^{1}$ The exposure to this constant load on the craniovertebral extensor muscles and the noncontractile structures causes a change in the biomechanical movement, and this increased stress can cause musculoskeletal damage or pain. ${ }^{1}$ The malalignment has been advocated to increase stress on the posterior cervical elements, affect the length---tension relationship in the cervical muscles, increase muscular activity level, restrict neck movement and impair cervical proprioception. ${ }^{4}$ This change in posture can lead to a spatial change between the spine and the line of gravity, causing an overload on muscles and connective tissues. ${ }^{1}$ Moreover, it was reported that extended periods of FHP can result in a decreased number of sarcomeres, as well as shortening of the muscle fibers, which can affect muscular contraction. ${ }^{1}$ The most potential impairment in neuromuscular functions occurring as a result of FHP include impaired cervical proprioception, weakened cervical muscles, and altered limits of stability. ${ }^{5}$ Photogrammetry, a simple and objective technique for measuring posture has shown good validity to examine craniovertebral posture. ${ }^{3}$ The craniovertebral angle (CVA) technique is one of the most common technique for evaluating the FHP which examines head status relative to the seventh cervical vertebrae $(\mathrm{C} 7)^{3}{ }^{3}$ Measurement of the craniovertebral (CV) angle is used as an index of the degree of head protraction in natural head posture (NHP). ${ }^{6}$ Smaller CV angles indicate greater protraction of the head and larger angles are more representative of 'ideal' sagittal plane head / neck alignment. ${ }^{6}$ Proprioception from the muscles is a primary sensory mechanism for motor control. ${ }^{7}$ Mechanoreceptors are the specialized nerve endings that provide sensory information in the form of proprioception. $^{8}$ Mechanoreceptors are transducers that convert mechanical stimuli into action potentials for transmission to the CNS. $^{8}$ During movements proprioception has importance for: feedback (reactive) control, feedforward (preparatory) control and the regulation of muscle stiffness, to achieve specific roles for movement acuity, joint stability, co-ordination and balance. ${ }^{8}$ Cervical proprioceptive information also has a highly important specific role for head and eye movement control. ${ }^{8}$ Abnormal Joint Position Error (JPE) has been tested in patients using 2 types of tests: (a) ability to actively relocate the head to the neutral position after it has been passively displaced and (b) ability to actively relocate the head to the reference position within a movement plane (Head Repositioning Accuracy [HRA]). ${ }^{9}$ The HRT is an inexpensive and simple method allowing quantification of head to trunk repositioning for various head attitudes and anatomical planes (i.e., transverse, sagittal). ${ }^{10}$ Head repositioning test have good reliability (ICC,0.81) and validity. ${ }^{11}$ In addition, authors reported a proprioceptive threshold, for defining kinesthetic alteration, set at $4.5^{\circ} .{ }^{10}$

Neck Retractions are one of the numerous techniques used by physical therapist to assess and treat patients with neck pain. ${ }^{12}$ This maneuver is advocated by McKenzie as an assessment and patient selftreatment technique aimed at increasing cervical range of motion, decreasing patient's pain and assisting in prevention of recurrences. ${ }^{12}$ Repeated cervical retraction can improve the postural alignment of FHP by strengthening the cervical muscles. ${ }^{13}$ Chin tuck as a static exercise, is known to increase the strength of cervical extensors and flexibility of cervical flexors. ${ }^{14}$

Muscle Energy Technique (MET) are classes of soft tissue osteopathic manipulation methods that incorporate precisely initiated, directed and controlled, patient initiated, isometric and/ or isotonic 

on cervical joint position sense in individuals with forward head posture.

contractions designed to improve musculoskeletal function and reduce pain. ${ }^{15,16}$ Post-isometric Relaxation (PIR) refers to the assumed effect of reduced tone experienced by a muscle, or a group of muscles, after brief periods following an isometric contraction. The basic principle behind PIR lies that after a muscle is contracted, it is automatically in a relaxed state for a brief, latent period. ${ }^{15,16}$ Muscle energy technique (MET) is a method of treatment that involves the voluntary contraction of subject's muscles in a precisely controlled direction, against a counterforce. MET is commonly useful method for achieving tonus release (inhibition) in a muscle. ${ }^{16,17}$ The craniocervical junction is the most mobile part of the spine where Suboccipital muscle (SOM) lies in the depth and connects and moves the head joints. ${ }^{18}$ The SOM has a high density of muscle spindle per gram SOM also shows a higher density of rotational muscles, in this case the obliquus inferior (OCI) and the superior (OCS).${ }^{18} \mathrm{The}$ fibre distribution of the SOM is very homogeneous, allowing both postural control and dynamic functions. ${ }^{18,19}$

\section{MATERIALS AND METHODS}

Ethical Committee approval was taken. A comparative study where 60 subjects (both genders) in the age group of 20-40 years having forward head posture (based on CVA assessment using AutoCAD software) were recruited using purposive sampling and randomly allocated to Group A $\quad(n=30)$ where Cervical Retraction exercise were given and Group B $(n=30)$ where Muscle Energy Technique was given. $^{3,20}$ Proprioceptive exercises were given to both the groups. The inclusion criteria for the study included subjects having forward head posture (asymptomatic) and having Craniovertebral angle between $40^{\circ}-60^{\circ}$ (using AutoCAD software). ${ }^{3,21,22}$ Subjects having neck pain, trauma (recent), neurological or inflammatory conditions, vestibular involvement were excluded. The outcome measure of the study was Cervical Joint position sense assessed using Head Repositioning Accuracy test. . $^{23,24,25}$

\section{PROCEDURE}

A written consent was taken from all the subjects in the language best understood by them. Selection of the subjects was done as per the inclusion and exclusion criteria. Purpose of the study and procedure were explained to the subjects prior to the study. Demographic data was noted down. Pre and Post (2 weeks) values of cervical joint position sense of physiological movements (flexion, extension, right rotation and left rotation) was assessed using head repositioning accuracy test.

Proprioception training: Exercises for proprioceptive intervention included head relocation practice, gaze stability, eyefollow and eye/head coordination exercises. Relocation practice involved the practice of relocating the head back to the natural head posture (neutral) and to predetermined positions in range, first with eyes open using feedback from a laser attached to their head and then with eyes closed. All active physiological movements of the cervical spine (flexion, extension, left rotation and right rotation) were used. Oculomotor exercises were progressed through several stages, initiating with eye movement with the head stationary, followed by movements of the head with visual fixation on a target. Third stage included Eye/head coordination exercises which commenced with rotation of the eyes and head to the same side, in both left and right direction. Finally, the patient practiced leading with the eyes first to a target, followed by the head, ensuring the eyes keep focused on the target. Both Group A and B received proprioception training of 10 counts for each exercise for 2 weeks- 3 sessions per week. ${ }^{9,24,25,26,27}$ 


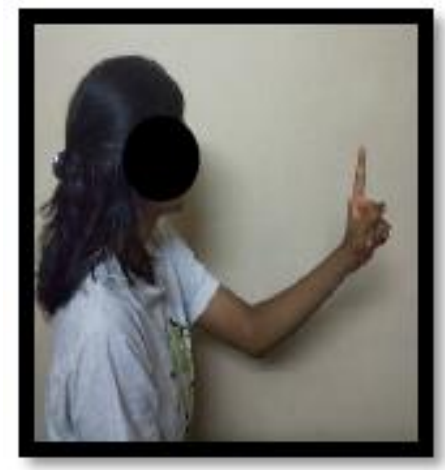

1. Eye movements with head stationary.

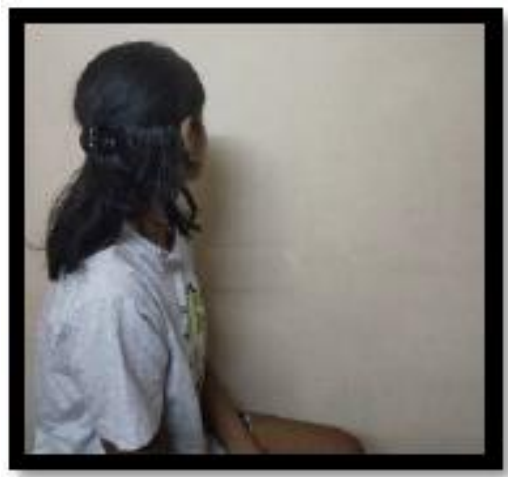

3. Movement of eyes and head to same side.

Cervical Retraction exercise (GROUP A): Group A were given Cervical retraction exercises (along with proprioception exercise) in the following manner. Subject performed 10 repetitions with hold for 10 counts thrice a week for 2 weeks. 1 .

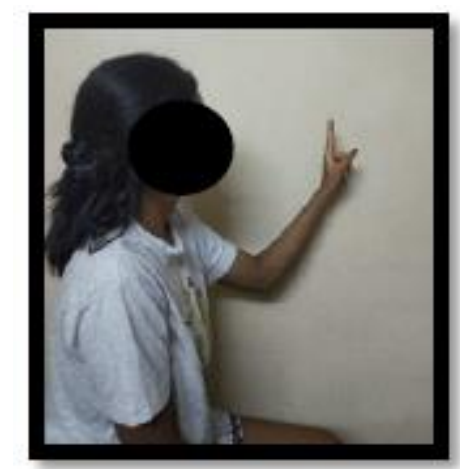

2. Movement of head with visual fixation.

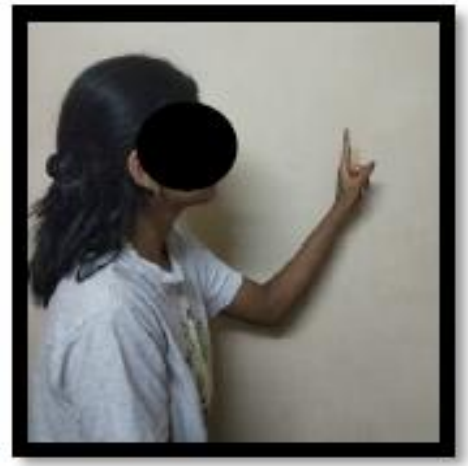

4. Eyes leading first to target followed by head.

Exercise was initiated in supine position and retraction exercise was performed with no lifting of head off the surface. 2. Exercise was followed by head retraction exercise in sitting. 3. Then followed by the same exercise in standing with wall support. ${ }^{28,29}$
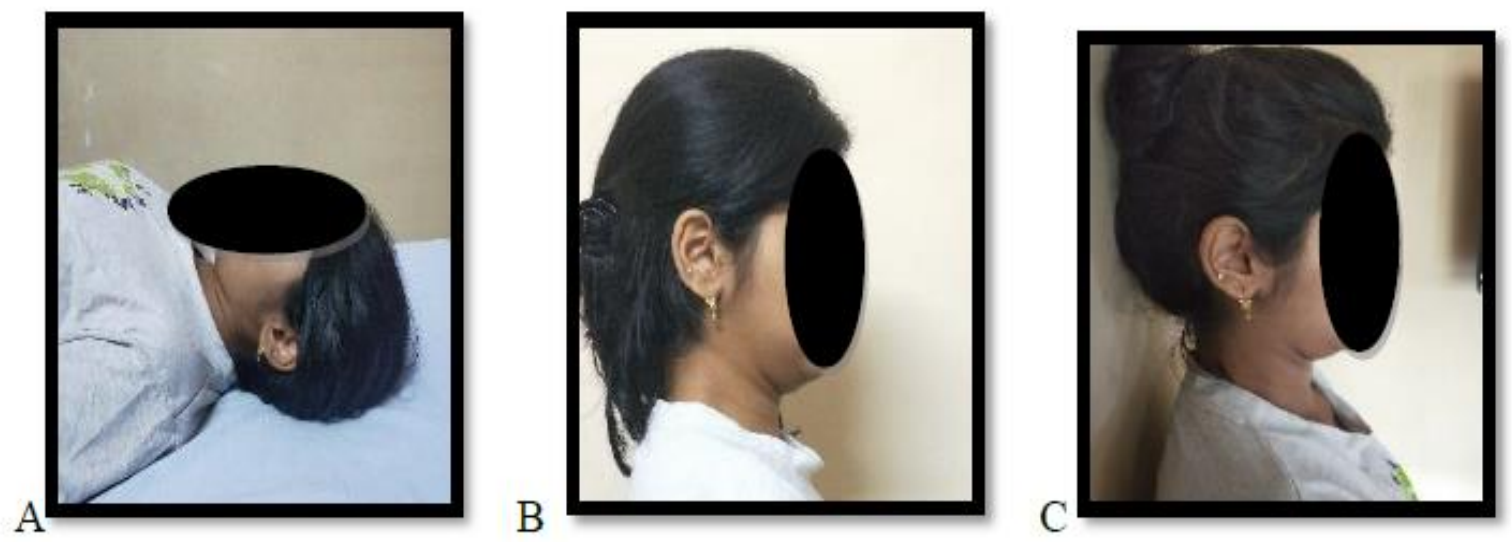

Cervical Retraction in A. Supine B. Sitting C. Standing

Muscle Energy Technique [MET] (GROUP B): Lewit's Post isometric relaxation technique of MET for Suboccipital muscle was given in supine position. Subjects performed 3 repetitions thrice a week for 2 weeks. Method: For suboccipitalis, therapist had to move neck in to flexion just short of cranioflexion barrier and Subject was asked to gently push back into craniocervical extension with mild effort (20\% of the maximum strength) for 10 seconds. . $^{16,28,30}$ 


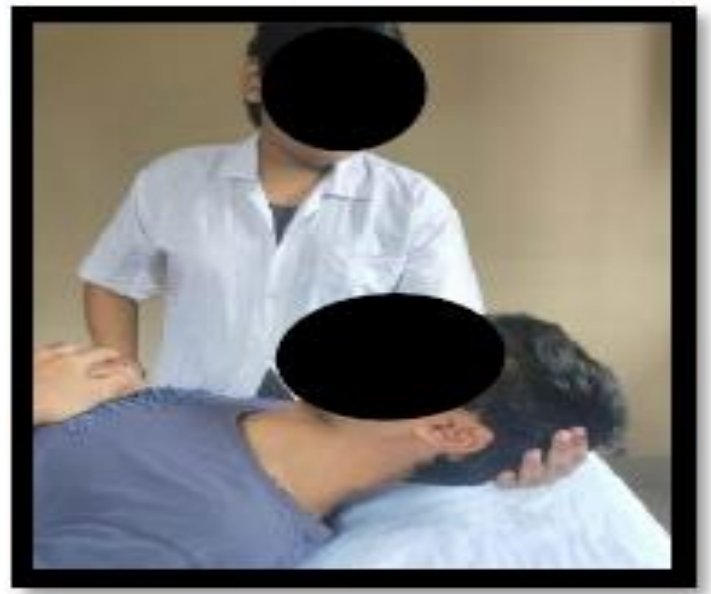

Muscle Energy Technique of Suboccipital Muscle in Supine Position.

\section{STATISTICAL ANALYSIS}

The data was entered using Microsoft Office Excel 2016 and was analysed using SPSS IBM trial version. The numerical data was analysed for normality using Kolmogorov-Smirnov test. Descriptive data analysis was done for Age and Cranio-vertebral angle and Mean and Standard Deviation was calculated. Since the data passed normality test, the data was analysed using Parametric tests. Paired t test was used to assess difference in cervical joint position sense between pre and post of both Group A and Group B. Unpaired t test was used to assess difference in cervical joint position sense between Group A and Group B. P value less than 0.05 was considered statistically significant.

\section{RESULT}

Within the group comparison for Group A (Cervical Retraction)

\begin{tabular}{|l|l|l|l|l|}
\hline & MEAN & SD & P VALUE & SIGNIFICANCE $(\mathbf{P}<\mathbf{0 . 0 5})$ \\
\hline FLEXION & 4.64 & \pm 1.52 & 0.000 & Significant \\
\hline EXTENSION & 5.56 & \pm 1.23 & 0.000 & Significant \\
\hline LEFT ROTATION & 3.48 & \pm 1.17 & 0.000 & Significant \\
\hline RIGHT ROTATION & 3.67 & \pm 1.02 & 0.000 & Significant \\
\hline
\end{tabular}

Within the group comparison for Group B (Muscle Energy Technique)

\begin{tabular}{|l|l|l|l|l|}
\hline & MEAN & SD & P VALUE & SIGNIFICANCE $(\mathbf{P}<\mathbf{0 . 0 5})$ \\
\hline FLEXION & 2.73 & \pm 0.60 & 0.000 & Significant \\
\hline EXTENSION & 2.65 & \pm 1.63 & 0.000 & Significant \\
\hline LEFT ROTATION & 1.65 & \pm 0.83 & 0.000 & Significant \\
\hline RIGHT ROTATION & 1.61 & \pm 1.45 & 0.000 & Significant \\
\hline
\end{tabular}

Between the group comparison (Group A vs Group B)

\begin{tabular}{|l|l|l|l|}
\hline & MEAN DIFFERENCE & P VALUE & SIGNIFICANCE $(\mathbf{P}<\mathbf{0 . 0 5})$ \\
\hline FLEXION & -1.73 & 0.000 & Significant \\
\hline EXTENSION & -1.93 & 0.000 & Significant \\
\hline LEFT ROTATION & -1.14 & 0.002 & Significant \\
\hline RIGHT ROTATION & -1.50 & 0.000 & Significant \\
\hline
\end{tabular}

The following major results were identified:

* There is statistically significant reduction in error of cervical joint position sense within the Group A (Cervical retraction) and Group B (Muscle Energy Technique) post intervention of 2 weeks.

* There is statistically significant reduction in error of cervical joint position sense between the Group A and Group B (Cervical Retraction and Muscle Energy Technique) post intervention of 2 weeks.

\section{DISCUSSION}

The present study was undertaken to assess and compare cervical retraction exercise and muscle energy technique on cervical joint position sense in individuals with forward head posture. Study was conducted on total 60 subjects having forward head posture. Study included individuals aged between 20-40 years (Group A (30.30 \pm 5.76) Group B (30.17 $\pm 5.98)$ ) and Craniovertebral Angle (Group A (45.12 \pm 1.17$)$ Group B (45.42 \pm 0.81$))$. In the study, Group A had $63 \%$ male and $37 \%$ 
female while Group B had $47 \%$ male and $53 \%$ females.

There is statistically significant difference $(\mathrm{p}<0.05)$ in cervical joint position sense within the Group A (Cervical retraction) and Group B (Muscle Energy Technique) post intervention of 2 weeks. However, Cervical Retraction exercise (Group A) is better as compared to Muscle Energy technique (Group B) in improving Cervical Joint Position Sense in individuals with Forward Head Posture.

Proprioceptive sensations provide feedback of the motor output and are vital in order to maintain the control of voluntary movement. ${ }^{31}$ Proprioception and motor control are interlinked that, it is often impossible to separate where the sensory signals subserve a direct motor function and where the same signals evoke proprioceptive sensations which might be used in control of movement. Proprioceptive afferents have roles in reflex responses at the spinal and cortical levels and provide feedback that allows the control of all purposeful movements. ${ }^{31}$

This can be explained by:

$\approx$ The muscle spindle density in the deep cervical muscles allows great precision of movement and also provides the proprioceptive information necessary for the control of eye-head-neck coordination and for the control of head movement and position. ${ }^{32}$

$\approx$ Altered afferent information from the visual, vestibular and cervical proprioceptive afferents can cause a loss of coordination between the head, eyes and upper limbs, affecting head orientation in space and relative to the trunk. ${ }^{32}$

$\approx$ The relocation practice in the proprioceptive training program directly trained the impairment and the outcome measure of Joint Position Error or because the program addressed the cervical afferent input in its functional role by the inclusion of eye movement exercises, noting the close relationship between the deep cervical extensors/rotators and horizontal eye movement. ${ }^{24}$

The findings of the study are in correlation with Gwendolen Jull et al (2007). They investigated the effect of two exercise regimes on cervical joint position sense. A significant pre to post intervention decrease in Joint position error was identified for both the proprioceptive training group $(\mathrm{p}<0.001)$ and the $\mathrm{C}-\mathrm{CF}$ training group $(\mathrm{p}<0.05)$. The results of this study indicate that both proprioceptive training and $\mathrm{C}-\mathrm{CF}$ training are efficacious in improving cervical JPE after a 6-week training period, although there were some marginally greater benefits with the proprioception training protocol. ${ }^{24}$ The results of the study are in alignment with critical review done by Cheryl Peterson et al (2013) and Barry Kim Humphreys et al (2002) that simple eye head neck coordination exercise is helpful in reducing functional impairment in terms of cervicocephalic kinesthesia. ${ }^{32,9}$

Cervical Retraction exercises have shown to improve Cervical Joint Position Sense The findings of this study are in accordance with findings of $\mathrm{G} V$ Krishna et al (2005) who had done the comparison between cervical retraction exercise versus isometric exercise on forward head posture. The results of the study showed that both these techniques show statistical significant difference in FHP posture, however cervical retraction was more beneficial.

This can be explained as follows:

$\approx$ Retraction of the head produces flexion in the upper segments and simultaneously causes extension in the lower segments of the cervical spine. It has been exhibited that more flexion occurs in upper cervical spine when the head is retracted than occurs when the head and neck are simply flexed. ${ }^{33}$

$\approx$ Cervical Retraction reverses any anterior shear or translation forces that may develop during prolonged end range positioning with head neck flexed or in protruded or forward head posture. ${ }^{33}$ 
$\approx$ This exercise stretches the structures that are adaptively shortened as result of long standing protruded head posture. ${ }^{14}$

$\approx$ Retraction (chin tuck) as a strengthening exercise has shown to improve deep anterior neck muscle force and torque by increasing the size of the muscle fibers. ${ }^{14}$

$\approx$ Also, flexion and retraction of upper cervical segments separates the $\mathrm{C} 2$ spinous process from the occiput which advocates that the suboccipital muscles will be elongated and thus may contribute to resisting end range neck retraction motion. ${ }^{12}$

$\approx$ Retraction of the head leads to flexion of the upper cervical segments which activates the deep cervical flexor musculature, which has been shown to have a relatively high density of muscle spindles. Thus, the repeated contractions involved in Craniocervical flexor (C$\mathrm{CF}$ ) training improves muscle spindle function translating to improved cervical proprioception. $^{24}$

$\approx$ Along with this, the improved cervical neuromuscular control gained from $\mathrm{C}$ $\mathrm{CF}$ training could decrease stresses placed on the joints and other structures of the cervical region. ${ }^{24}$

$\approx$ Abnormal joint stress may alter firing of cervical afferents with resultant changes in proprioceptive function. ${ }^{37}$ Thus, changes in activity of the deep and superficial muscles may be responsible for changes in proprioception. It has been proven that, due to FHP the force generated my Sternocleidomastoid (SCM) and Splenii muscles are reduced as compared to normal head posture. Hence, Retraction helps to realign the head in normal posture, thereby improving the force production in sternocleidomastoid and splenii muscle attached to lower segments of cervical spine. $^{34}$

Similarly studies done by Meysham Goosheh et al (2018), Kyeong-Jin Lee et al (2015) comply with our study findings. ${ }^{14,34}$
Muscle Energy Technique has shown to reduce error in cervical joint position sense which can be explained as:

$\approx$ MET induces relaxation of hypertonic musculature and, where appropriate, the subsequent stretching of the muscle. It has influence on both static and kinetic posture, because of the effects on proprioceptive and interoceptive afferent pathways. ${ }^{16,19}$

$\approx$ Muscle spindles have been recognized to play a major role in increasing muscle tone and in the pathophysiology of muscle pain syndromes. The deep neck muscles (suboccipital) have an extremely high muscle spindle density almost five times higher than that of the splenius capitis and three times that of the semispinalis capitis muscle. ${ }^{19}$

$\approx$ The high muscle spindle density and the special features of the muscle spindles in the deep neck muscles allow not only great precision of movement but also adequate proprioceptive information needed both for control of head position and movements and for eye/head movement. ${ }^{19}$

$\approx$ The morphologic and biomechanical properties of Suboccipital muscles (Rectus Capitis Posterior) seem to be optimized to contribute to segmental stability of the Occipito-Atlantal and Atlanto-Axial joints as a function of head position through control of the angular position of the posterior arch of the atlas and the posterior process of the axis relative to the occiput and to one another. ${ }^{18}$

$\approx$ MET and related post-isometric techniques diminish pain and discomfort when applied to the spine or muscles by involving central and peripheral modulatory mechanisms, such as activation of muscle and joint mechanoreceptors that involve centrally mediated pathways, like the periaqueductal grey (PAG) in the midbrain, or nonopioid serotonergic and noradrenergic descending inhibitory pathways. $^{35}$ 
$\approx$ MET produces joint motion while actively recruiting muscles, which has an effect on proprioceptive feedback, motor control, and motor learning. ${ }^{35}$

The findings of this study are in accordance with findings of Edrish Saifee Contractor et al, (2019). Muscle Energy Technique decreases hyperactivation and tightness in shortened deep cervical extensors in subjects with FHP. The mechanism behind this result is neurophysiologic mechanism that it activated Golgi Tendon Reflex, inhibits the alpha motor neuron and thereby inhibited Suboccipital muscles. ${ }^{30}$

There is statistically significant difference within the group, however from the statistics we can infer that Cervical Retraction is better than Muscle Energy Technique in improving Cervical Joint Position Sense in individuals with Forward Head Posture. The probable causes for this can be: cervical retraction exercise leads to the strengthening of deep neck flexors, along with stretching of suboccipital muscles and activation of muscles in the lower cervical spine like splenii along with sternocleidomastoid while MET was only given to suboccipital muscle.

\section{CONCLUSION}

that:

The findings of the study conclude

* Cervical Retraction exercise can be used to improve Cervical Joint Position Sense in individuals with Forward Head Posture.

* Muscle Energy technique can be used to improve Cervical Joint Position Sense in individuals with Forward Head Posture.

* However, Cervical Retraction exercise is better as compared to Muscle Energy technique in improving Cervical Joint Position Sense in individuals with Forward Head Posture.

\section{CLINICAL IMPLICATION}

* Proprioception is important for feedback and feedforward control, regulation of muscle stiffness, to achieve specific roles for movement acuity, joint stability, co-ordination and balance, which is affected in individuals with forward head posture, hence it is vital to improve joint position sense.

* Cervical retraction exercise as well as proprioceptive exercise once mastered can be performed by the patients themselves as a home program for improving joint position sense.

\section{ACKNOWLEDGEMENTS}

I express my deep sense of gratitude and sincere thanks to our sir Dr Ajay Kumar and my guide Dr Parag Kulkarni for their encouragement, guidance and constant supervision throughout the study. Also, my heartfelt thanks to all the subjects who willingly participated in the study and cooperated with me.

\section{Conflict of Interest: None}

\section{Source of Funding: None}

\section{Ethical Approval: Approved}

\section{REFERENCES}

1. Kim DH, Kim CJ, Son SM. Neck pain in adults with forward head posture: effects of craniovertebral angle and cervical range of motion. Osong Public Health and Research Perspectives. 2018 Dec;9(6):309.

2. S Sannakki S. Correlation Of Neck Column Length With Craniovertebral And Craniohorizontal Angles In Normal Individuals (Doctoral dissertation). 2010

3. Salahzadeh Z, Maroufi N, Ahmadi A, Behtash H, Razmjoo A, Gohari M, Parnianpour M. Assessment of forward head posture in females: observational and photogrammetry methods. Journal of back and musculoskeletal rehabilitation. 2014 Jan 1;27(2):131-9.

4. Ghamkhar L, Kahlaee AH. Is forward head posture relevant to cervical muscles performance and neck pain? A case-control study. Brazilian journal of physical therapy. 2019 Jul 1;23(4):346-54.

5. Migliarese S, White E. Review of ForwardHead Posture and Vestibular Deficits in 

on cervical joint position sense in individuals with forward head posture.

Older Adults. Current Geriatrics Reports. 2019 Sep 15;8(3):194-201.

6. Kerry C. Reliability of measuring natural head posture using the craniovertebral angle. Irish Ergonomics Review. 2003; 2003:37.

7. Comerford MJ, Mottram SL. Movement and stability dysfunction-contemporary developments. Manual therapy. 2001 Feb $1 ; 6(1): 15-26$.

8. Röijezon U, Clark NC, Treleaven J. Proprioception in musculoskeletal rehabilitation. Part 1: Basic science and principles of assessment and clinical interventions. Manual therapy. 2015 Jun 1;20(3):368-77.

9. Humphreys BK. Cervical outcome measures: testing for postural stability and balance. Journal of manipulative and physiological therapeutics. 2008 Sep 1;31(7):540-6.

10. Dugailly PM, De Santis R, Tits M, Sobczak $S$, Vigne A, Feipel V. Head repositioning accuracy in patients with neck pain and asymptomatic subjects: concurrent validity, influence of motion speed, motion direction and target distance. European Spine Journal. 2015 Dec 1;24(12):2885-91.

11. Pinsault N, Fleury A, Virone G, Bouvier B, Vaillant J, Vuillerme N. Test-retest reliability of cervicocephalic relocation test to neutral head position. Physiotherapy theory and practice. 2008 Jan 1;24(5):38091.

12. Pearson Neil, Walmsley Roy. Trial into the effects of repeated neck retractions in normal subjects. Spine. 1995. Volume 20, number 11, pp 1245-1251.

13. Han JW, Kim KH, Bae TS, Blaikie K. Biomechanical Analysis of Chin Tuck Exercise with a Subject-Specific Neck Model for the Forward Headed. International Journal of Precision Engineering and Manufacturing. 2018 Apr 1;19(4):587-92.

14. Goosheh M, Shafizadegan Z, Rezaieian ZS, Salamehzadeh F, Baharlouei H. Comparing the immediate effect of chin tuck and turtle exercises on forward head posture: A single blind randomized clinical trial. Polish Annals of Medicine. 2019 Jan 1;26(1).

15. Toshniwal P, Amarnatha TK. To Study the Effect of Muscle Energy Technique of Pectoral Muscle on Neck Pain and Cervical Range of Motion in Individuals with
Forward Head Posture-An Experimental Study. International Journal of Health Sciences and Research. 2019;9(9):19-25.

16. Chaitow L. Muscle energy techniques. 2nd Edition

17. Kumari C, Sarkar B, Banerjee D, Alam S, Sharma R, Biswas A. Efficacy of muscle energy technique as compared to proprioceptive neuromuscular facilitation technique in chronic mechanical neck pain: a randomized controlled trial. Int $\mathrm{J}$ Health Sci Res. 2016;6:152-61.

18. Reinhardt R. Suboccipital MusculatureMorphology, Functions and Variants-An Update.

19. Liu JX, Thornell LE, Pedrosa-Domellöf F. Muscle spindles in the deep muscles of the human neck: a morphological and immunocytochemical study. Journal of Histochemistry \& Cytochemistry. 2003 Feb;51(2):175-86.

20. Sullivan L. Power and sample size determination. 2017.

21. Lee MY, Lee HY, Yong MS. Characteristics of cervical position sense in subjects with forward head posture. Journal of physical therapy science. 2014; 26(11):1741-3.

22. Lee HS. The Analysis of severity of forward head posture with observation and photographic method. Journal of Korean Society of Physical Medicine. 2015 Aug 31;10(3):227-35.

23. Mugdha O, Kotecha Dhara J, Sujata Y. Assessment and Comparison of cervical Joint position sense in subjects with chronic neck pain vs normals. Int $\mathrm{J}$ Physio [Internet]. 2015Jun.7 [cited 2020Aug.29];2(3):483-6.

24. Jull G, Falla D, Treleaven J, Hodges P, Vicenzino B. Retraining cervical joint position sense: the effect of two exercise regimes. Journal of Orthopaedic Research. 2007 Mar;25(3):404-12.

25. Clark NC, Röijezon U, Treleaven J. Proprioception in musculoskeletal rehabilitation. Part 2: Clinical assessment and intervention. Man Ther. 2015;20(3):378-387

26. Revel M, Minguet M, Gergoy P, Vaillant J, Manuel JL. Changes in cervicocephalic kinesthesia after a proprioceptive rehabilitation program in patients with neck pain: a randomized controlled study. 
Isha Shah et.al. Assessment and comparison between cervical retraction exercise and muscle energy technique on cervical joint position sense in individuals with forward head posture.

Archives of physical medicine and rehabilitation. 1994 Aug 1;75(8):895-9.

27. Treleaven J. Sensorimotor disturbances in neck disorders affecting postural stability, head and eye movement control. Man Ther. 2008;13(1):2-11.

28. Narang Sakshi, Mehra Suman, Sikka Geetanjali. Effect of Muscle Energy Technique and Deep Neck Flexors Exercise on Pain, Disability and Forward Head Posture in Patients with Chronic Neck Pain. Indian Journal of Physiotherapy and Occupational Therapy - An International Journal January 2014 8(4):43

29. Jung YW. Effects of McKenzie Exercise on the Functional Recovery and Forward Head Posture of Chronic Neck Pain Patients. Journal of Korean Society of Physical Medicine. 2006 May 31;1(1):93-108

30. Contractor ES, Shah S, Dave P. To Study the Immediate Effect of Suboccipital Muscle Energy Technique on Craniovertebral Angle and CranioHorizontal Angle on Subjects with Forward Head Posture. International Journal of Health Sciences and Research. 2019; 9(3):83-7.

31. Bokaee F, Rezasoltani A, Manshadi FD, Naimi SS, Baghban AA, Azimi $H$. Comparison of cervical muscle thickness between asymptomatic women with and without forward head posture. Brazilian journal of physical therapy. 2017 May 1;21(3):206-11.

32. Petersen CM, Zimmermann CL, Tang R. Proprioception interventions to improve cervical position sense in cervical pathology. International Journal of Therapy and Rehabilitation. 2013 Mar 2;20(3):15463.

33. McKenzie R A. The cervical and thoracic spine, mechanical diagnosis and therapy. Pg 118

34. Lee KJ, Han HY, Cheon SH, Park SH, Yong MS. The effect of forward head posture on muscle activity during neck protraction and retraction. Journal of physical therapy science. 2015;27(3):977-9.

35. Fryer G. Muscle energy technique: An evidence-informed approach. International Journal of Osteopathic Medicine. 2011 Mar $1 ; 14(1): 3-9$.

How to cite this article: Shah I, Kulkarni P, Kumar A. Assessment and comparison between cervical retraction exercise and muscle energy technique on cervical joint position sense in individuals with forward head posture. International Journal of Science \& Healthcare Research. 2022; 7(1): 199-208. DOI: https:// doi.org/10.52403/ijshr.20220132 